\title{
Peculiarities of a magnetic transition in a quasi-one-dimensional ferromagnet $\mathrm{PbMnBO}_{4}$
}

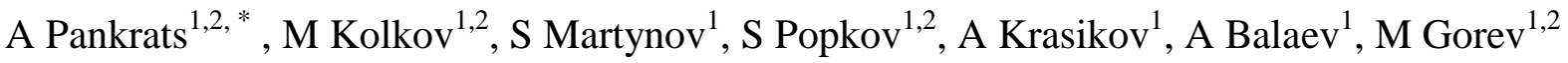 \\ ${ }^{1}$ Kirensky Institute of Physics, Federal Research Center KSC SB RAS, Krasnoyarsk, Russia \\ ${ }^{2}$ Siberian Federal University, Krasnoyarsk, Russia
}

* Corresponding author, E-mail address: pank@iph.krasn.ru

\begin{abstract}
Near the Curie temperature $T_{C}=30.3 \mathrm{~K}$, the temperature dependences of the magnetization and heat capacity of a single-crystal ferromagnet $\mathrm{PbMnBO}_{4}$ in the magnetic fields of $1,3,10$ and $30 \mathrm{kOe}$ are studied. In the strong magnetic fields, both the magnetic contribution to the specific heat and the nonlinearity of the field dependences of the magnetization are maintained up to the temperatures exceeding $T_{C}$ more than twice. It is assumed that in $\mathrm{PbMnBO}_{4}$ the difference between $T_{C}$, the paramagnetic Curie temperature $\theta=49 \mathrm{~K}$ and the broad temperature region above $T_{C}$ where the magnetic contribution to the specific heat is significant is due to the quasi-one-dimensional character of the magnetic structure of this ferromagnet. Using both the estimation of $T_{C}$ from the Ginzburg-Landau field theory and the $\theta$ value, the total exchange interaction parameters $2 J \approx 40.4 \mathrm{~K}$ (intrachain) and $z^{\prime} J \approx 8.8 \mathrm{~K}$ (interchain) are determined, with $z^{\prime}=$ 4 being the number of neighboring chains. The estimation shows that the Ginzburg-Landau field theory describing the quasi-one-dimensional behavior of $\mathrm{PbMnBO}_{4}$ is well applicable in the temperature range from to $T=S^{2} J \approx 80 \mathrm{~K}$. Above this temperature, the mean field approximation with the exchange parameter $\lambda_{\theta}$ based on the paramagnetic Curie temperature $\theta$ describes well the experimental temperature dependences of the magnetization in the strong magnetic field and the specific heat is determined by the lattice contribution.
\end{abstract}

\section{Introduction.}

The search for and synthesis of new magnetic materials, as well as the investigation of their physical properties, are the priority tasks in solid-state physics. Such studies allow one to find promising materials for technical applications, and also provide experimental data for studying new physical effects arising at the intersection of magnetic, electrical, elastic, and other properties of solid state physics. The crystal classes which admit the existence of a family of isomorphous magnets with different magnetic ions in one of the crystallographic positions attract particular interest. This property can lead to a variety of physical properties, in particular, to different magnetic structures in the family of isomorphous crystals.

Orthoborate crystals with the general formula $\mathrm{PbABO}_{4}$ are representatives of such an interesting family. Their structure for $\mathrm{A}=\mathrm{Ga}, \mathrm{Al}$ was first studied by $\mathrm{H}$. Park et al. [1] where the orthorhombic structure with the space group Pnma was established. Later the same group of authors investigated the magnetic properties of polycrystalline compounds with $\mathrm{A}=\mathrm{Fe}, \mathrm{Cr}$, and Mn [2] and found that the type of magnetic order depends on the paramagnetic ion A. The antiferromagnetic order was established for $\mathrm{Fe}$ and $\mathrm{Cr}$ with the Néel temperatures of $125 \mathrm{~K}$ and 8.3 $\mathrm{K}$, respectively. The compound with $\mathrm{A}=\mathrm{Mn}$ showed the ferromagnetic order with the Curie temperature $T_{C}=31 \mathrm{~K}$. 
Magnetic studies on single crystals have a number of advantages. Single crystals have a higher quality than polycrystalline samples which often contain extraneous phases. It is also important that the studies on single crystals provide more information allowing one to study the anisotropic properties of matter. The magnetic studies of the $\mathrm{PbFeBO}_{4}$ single crystals which were grown by spontaneous crystallization allowed us [3] to explain them as typical of the threedimensional antiferromagnetic order and to refine the Neel temperature as $T_{N}=115 \mathrm{~K}$. We also investigated the dielectric properties of $\mathrm{PbFeBO}_{4}$ and found anomalies for both polycrystalline and single-crystal samples, which indicates the correlation between the magnetic and electrical subsystems in the crystals.

The $\mathrm{PbMnBO}_{4}$ single crystals were also first grown using spontaneous crystallization by a flux technique [4]. The analysis of its magnetic and resonance properties showed [4] that the static Jahn-Teller effect characteristic of the $\mathrm{Mn}^{3+}$ ion leads to the ferromagnetic exchange interaction in the $\mathrm{PbMnBO}_{4}$ crystal. The same effect apparently causes the strong magnetic anisotropy in the crystal. The significant effective anisotropy fields of the crystal determine the energy gap in the FMR spectrum which is extraordinarily large for ferromagnets ( $112 \mathrm{GHz}$ at $\mathrm{T}=4.2 \mathrm{~K}$ ). Another unusual property of the $\mathrm{PbMnBO}_{4}$ crystals was observed when studying the temperature dependences of the magnetization and resonance fields. In the external magnetic field, the induced ferromagnetic ordering is retained in the crystal above the Curie temperature up to the temperatures much higher than $T_{C}$. Thus, the aim of this work is to study in detail the transformation of the ferromagnetic state to a paramagnetic one in the presence of the external magnetic field.

A detailed study of the magnetic and thermophysical properties of $\mathrm{PbMnBO}_{4}$ shows that the nonlinearity of the field dependences of the magnetization inherent to the ordered ferromagnetic state and the magnetic contribution to the specific heat actually remains in this crystal up to temperatures exceeding more than twice the Curie temperature which is $30.3 \mathrm{~K}$ in accordance with the specific heat data. It is suggested that the main reason for this is the quasi-onedimensional character of the magnetic structure of the crystal. The parameters of intra- and interchain exchange interactions are estimated using the experimental values of the magnetic ordering temperature and paramagnetic Curie temperature. The peculiarity of the magnetic structure of $\mathrm{PbMnBO}_{4}$ is that these parameters differ significantly less than in classical quasi-onedimensional magnets. Above $\sim 80 \mathrm{~K}$, the specific heat of this ferromagnet is determined by the lattice contribution, and the magnetic properties are well described within the framework of the mean field theory with the exchange parameter calculated using the paramagnetic Curie temperature.

\section{Experimental details}

The studies were carried out on $\mathrm{PbMnBO} 4$ single crystals grown by spontaneous crystallization using the technique described in [4]. The magnetic measurements were performed using an original vibrating sample magnetometer in the temperature range from 4.2 to $300 \mathrm{~K}$ and magnetic fields of up to $70 \mathrm{kOe}$. PPMS-9 (Quantum Design) was used to measure the specific heat in the temperature range $2-300 \mathrm{~K}$ and in magnetic fields up to $30 \mathrm{kOe}$. 


\section{Experimental results}

To correctly compare the results of the magnetic and thermophysical studies, the temperature dependences of the magnetization and specific heat were measured in the same applied magnetic fields. Fig. 1 shows the temperature dependences of the magnetization measured in the temperature range $4.2-80 \mathrm{~K}$ in the magnetic field of 3, 10 and $30 \mathrm{kOe}$ applied in the direction of easy magnetization (the orthorhombic axis $a$ ). In the figure, the magnetization is given as normalized to the maximum in the experimental value of the magnetization measured at $\mathrm{T}=4.2 \mathrm{~K}$ and field of $70 \mathrm{kOe}$ (taken from our previous study [4]). At low temperatures, the magnetization dependence measured at $3 \mathrm{kOe}$ goes slightly below the other curves. Taking into account the field dependences of the magnetization [4], this feature can be explained by the fact that the magneti-

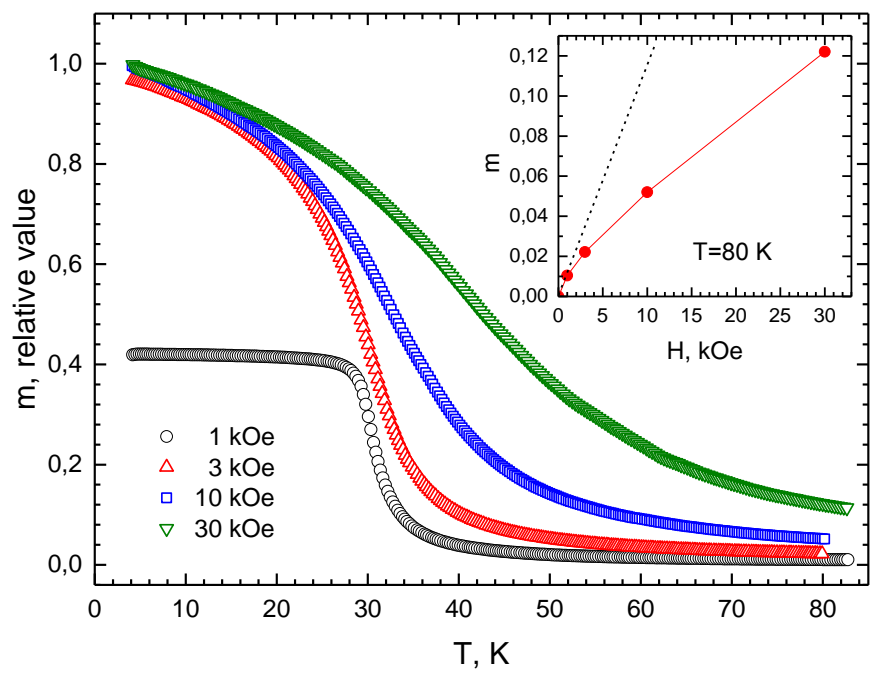

Fig. 1. Temperature dependences of the relative magnetization of $\mathrm{PbMnBO}_{4}$ measured in the easy direction in the magnetic fields of $1,3,10$ and $30 \mathrm{kOe}$. The inset shows the field dependence of the relative magnetization at $\mathrm{T}=80 \mathrm{~K}$.

zation is not yet fully saturated in this measuring field due to the domain structure.

The magnetization dependence measured in the field of $1 \mathrm{kOe}$ is also given here for comparison. An unusual form of this temperature dependence can easily be understood from the field dependences of the magnetization given in [4]. Due to the domain structure, saturation of the magnetization at low temperatures occurs in the field just above $3 \mathrm{kOe}$. The initial parts of the magnetization isotherms corresponding to the rearrangement of the domain structure in fields up to $1 \mathrm{kOe}$ coincide at all temperatures up to $\mathrm{T}=25 \mathrm{~K}$. Therefore, the magnetization remains almost constant at all temperatures below the Curie temperature and decreases sharply only with the approach to Tc.

The larger the measuring field, the slower the magnetization decreases at temperatures above $T_{C}$. As the inset in Fig. 1 shows, the field dependence of the magnetization based on the temperature dependences remains nonlinear even at $\mathrm{T}=80 \mathrm{~K}$. The direct measurements of the field dependences of the magnetization in $\mathrm{PbMnBO}_{4}$ given in [4] also demonstrate a nonlinear character at temperatures above $T_{C}$ up to the maximum in the experiment temperature of $77 \mathrm{~K}$.

The specific heat was also measured on the single crystal in the magnetic fields of $0,3,10$, and $30 \mathrm{kOe}$ (Fig. 2) applied in the orthorhombic axis $a$. In the absence of the field, the specific heat has a pronounced $\lambda$-peak at the Curie temperature whose refined value is $T_{C}=30.3 \mathrm{~K}$. In the presence of the magnetic field, as it should be, the jump of the specific heat disappears; the $\lambda$ peak transforms into a hump which broadens and shifts towards the higher temperatures as the magnetic field increases. 


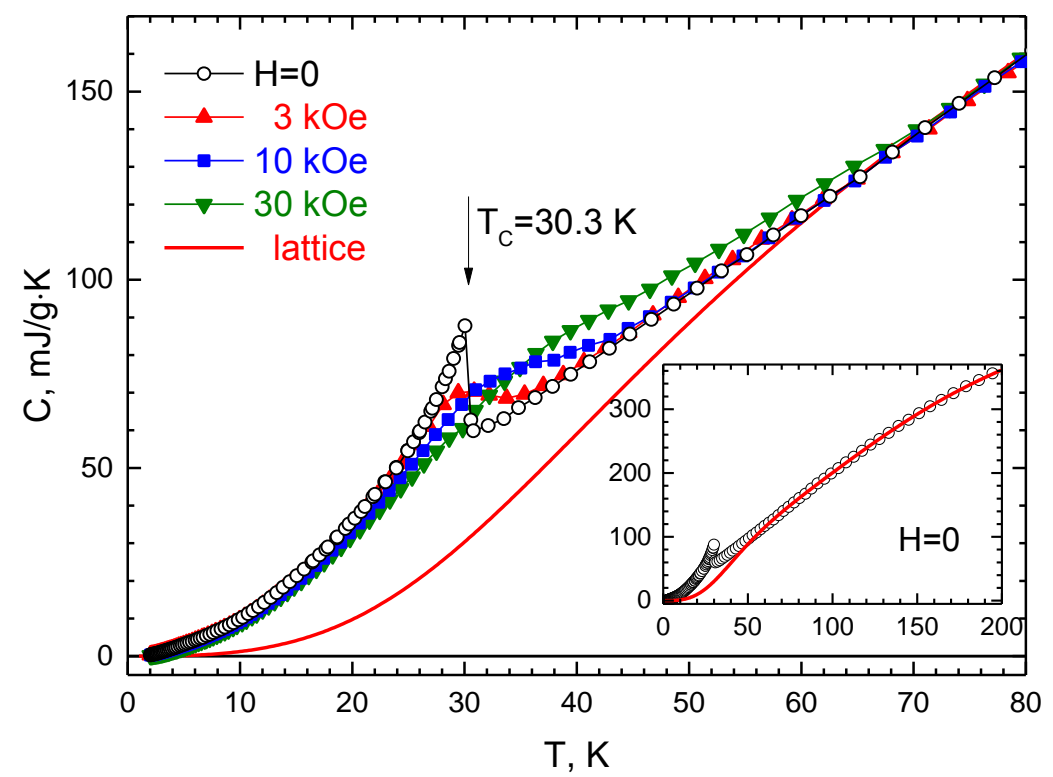

4. Discussion
Fig. 2. Temperature dependences of the specific heat measured in different magnetic fields

The Debye-Einstein model was used to calculate the contribution of the crystal lattice to the specific heat; this contribution is shown by the solid red line in Fig. 2. The Debye and Einstein temperatures are fitted as $\theta_{\mathrm{D}}=245.6 \mathrm{~K}$ and $\theta_{\mathrm{E}}=609.6 \mathrm{~K}$. The inset shows the temperature dependence of the specific heat measured in zero magnetic field in the temperature range up to $200 \mathrm{~K}$. It can be seen that the lattice contribution is decisive at high temperatures. In zero magnetic field, the deviation of the experimental data from the lattice contribution appears at temperatures slightly above $60 \mathrm{~K}$ and increases when approaching the Curie temperature. It is obvious that these deviations are caused by fluctuations due to the establishment of the magnetic order.

The experimental specific heat can be represented by the sum of the lattice $C_{\text {lat }}$ and magnetic $C_{m a g}$ contributions. The temperature dependences of the magnetic contribution to the specific heat are shown in Fig. 3. In the zero field, the magnetic contribution to the specific heat appears upon cooling below $65 \mathrm{~K}$ and increases abruptly at the Curie temperature. With the application of the magnetic field, this sharp jump transforms into a smooth hump which broadens and shifts towards higher temperatures with the increasing magnetic field.

The magnetic contribution to the specific heat allows one to calculate the entropy associated with the transition in the magnetic subsystem. It should be noted that for all magnetic fields the entropy tends to a level determined by the quantity $\Delta S_{\text {mag }}=R \ln (2 S+1)=39.71 \mathrm{~mJ} / \mathrm{g} \cdot \mathrm{K}$, confirming a purely magnetic character of the transition. But the rate of approaching this level depends on the applied magnetic field. Thus, in the zero magnetic field the relative contribution to the entropy of the transition at the Curie temperature reaches $\sim 0.8$ of the maximum level $\Delta \mathrm{S}$, and in the magnetic field of $30 \mathrm{kOe}$ it is only $\sim 0.6$. Accordingly, the maximum entropy of the transition in such a magnetic field reaches saturation at temperatures above $70 \mathrm{~K}$ while in the zero field this level is reached even at $60 \mathrm{~K}$. 


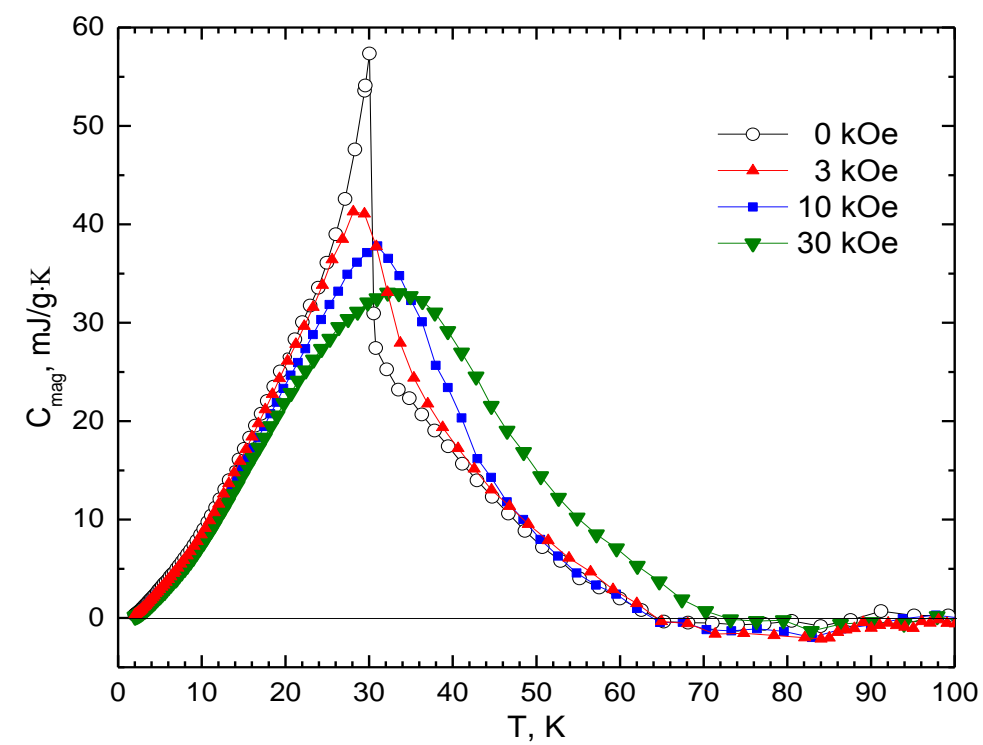

Fig. 3. Temperature dependences of the magnetic contribution to the specific heat measured in different magnetic fields.

Thus, both the thermophysical and magnetic properties of the $\mathrm{PbMnBO}_{4}$ crystal indicate that the field-induced ferromagnetic order is partially retained at temperatures much greater than $T_{C}$. However, even in the absence of the magnetic field some features of the ordered state also persist in a wide temperature range above $T_{C}$ Judging from the magnetic contribution to the specific heat (Fig. 3), this contribution even in the zero magnetic field vanishes only at $\mathrm{T} \approx 65 \mathrm{~K}$ which is twice as $T_{C}$. A detailed comparison of such contributions measured in the external magnetic fields shows that the relatively weak field ( 3 and $10 \mathrm{kOe}$ ) leads only to the smearing of the jump in the temperature range near $T_{C}$, and with further heating the temperature dependences of the magnetic contribution coincide with the curve for the zero field at temperatures above $\sim 40 \mathrm{~K}$ for $3 \mathrm{kOe}$ and $\sim 48 \mathrm{~K}$ for $10 \mathrm{kOe}$. But in the stronger field of $30 \mathrm{kOe}$, the magnetic contribution to the specific heat remains substantially higher than the curve for zero field at all temperatures above $T_{C}$ up to the disappearance of this contribution at $\mathrm{T} \approx 72 \mathrm{~K}$. The same features can also be noticed in the temperature dependence of the magnetic contribution to the entropy (Fig. 4).

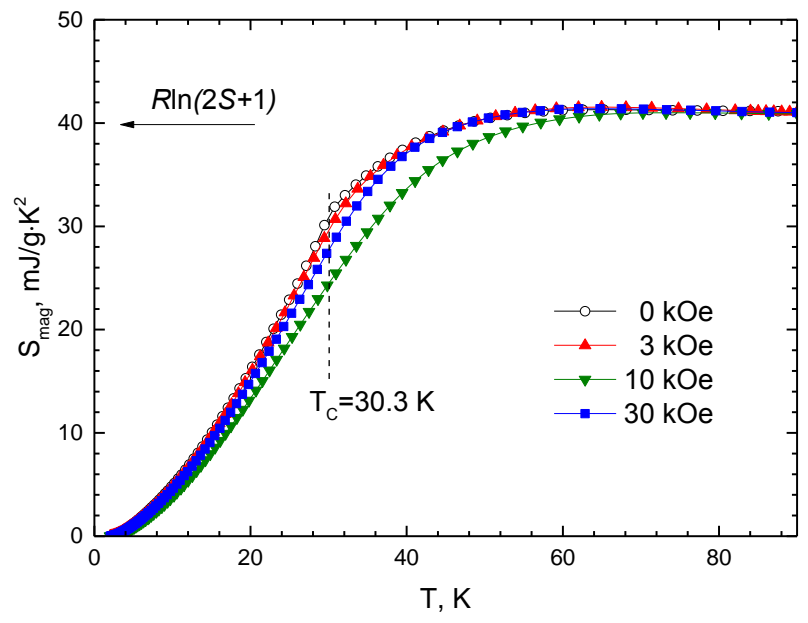

Fig. 4. Temperature dependences of the magnetic contributions to the entropy.

Another important peculiarity of the magnetic properties of $\mathrm{PbMnBO}_{4}$ is the considerable discrepancy between $T_{C}$ and the paramagnetic Curie temperature $\theta$ obtained from the high-temperature part of the magnetic susceptibility in the paramagnetic region. In $\mathrm{PbMnBO}_{4}$, the paramagnetic Curie temperature observed here for the direction of easy magnetization is equal to $\theta \approx 49 \mathrm{~K}^{1}$ exceeding $T_{C}$ by more than a factor of 1.5 .

In ferromagnetic metals and alloys, a similar temperature difference gave rise to the term "smeared magnetic transition" [5], the degree of smearing was usually charac-

${ }^{1}$ This value of the paramagnetic temperature $\theta$ is given for the orthorhombic axis $a$, for the other two axes the values are $51 \mathrm{~K}$ (axis $b$ ) and $47 \mathrm{~K}$ (axis $c$ ). All values were defined here more exactly than in [4] from the susceptibility data for $\mathrm{T}>175 \mathrm{~K}$. 
terized by the temperature interval $\Delta T_{s m}=\theta-T_{C}$. It is usually assumed that the short-range magnetic order is retained above $T_{C}$ within this interval due to the fluctuations of the magnetic order. One of the possible reasons of fluctuations in such compounds can be the inhomogeneities of the crystal structure. As an example, one can mention ferromagnetic nickel [5] with $T_{C}=631 \mathrm{~K}$ for which $\Delta T_{\mathrm{sm}} \approx 14 \mathrm{~K}$, with $\Delta T_{s m}<<T_{C}$ [5]. In metal alloys with a random distribution of atoms in the crystal structure, the value of $\Delta T_{s m}$ can reach $70 \mathrm{~K}$ [5] remaining anyway significantly lower than $T_{C}$. Obviously, the great difference between $T_{C}$ and $\theta$ in $\mathrm{PbMnBO}_{4}$ is of different physical nature which will be discussed below.

The comparison of the temperature dependence of the magnetization curves for $\mathrm{PbMnBO}_{4}$ in the external magnetic field $H_{0}$ directed along the easy axis of magnetization with the calculation in the framework of the mean field approximation was carried out on the basis of the Hamiltonian for the spin $S=2$ :

$$
H=g \mu_{B} H_{0} \sum_{i} S_{i}^{z}+\sum_{i>j} J_{i j} \mathbf{S}_{i} \mathbf{S}_{j}+K_{1} \sum_{i} S_{i}^{z 2}+K_{2} \sum_{i} S_{i}^{x 2},
$$

where the parameters of the two-axis anisotropy $K_{1}$ and $K_{2}$ (both $<0$ ) obtained from the FMR data [4] are used.

In this approximation, the relative magnetization of one ion has the form

$$
\begin{aligned}
& \frac{\left\langle S_{i}\right\rangle}{S}=\sigma=\frac{e^{-\beta K} \sinh \left(\beta g \mu_{B} H_{\mathrm{eff}}\right)+2 e^{-4 \beta K} \sinh \left(2 \beta g \mu_{B} H_{\mathrm{eff}}\right)}{0.5+e^{-\beta K} \cosh \left(\beta g \mu_{B} H_{\mathrm{eff}}\right)+2 e^{-4 \beta K} \cosh \left(2 \beta g \mu_{B} H_{\mathrm{eff}}\right)}, \\
& H_{\mathrm{eff}}=H_{0}+H_{E}, \mathrm{~K}=\mathrm{K}_{1}-\frac{\mathrm{K}_{2}}{2},
\end{aligned}
$$

where the exchange field has the form $H_{E}=\lambda S \sigma / g \mu_{B}$ and the secular part of the single-ion anisotropy $K / g \mu_{B}=-11 \mathrm{kOe}$ is taken into account. The non-secular part of the anisotropy of the Hamiltonian (1)

$$
H_{n s}=\frac{K_{2}}{4}\left(S^{+2}+S^{-2}\right)
$$

gives a small correction to the magnetization in the considered temperature and field regions and can be rejected.

The mean field coefficient $\lambda$ was determined from the experimental values of both the paramagnetic Curie temperature $\theta=49 \mathrm{~K}$ and the temperature of the ferromagnetic ordering $T_{C}=$ $30.3 \mathrm{~K}$ using the following expressions:

$$
\begin{aligned}
& \lambda_{\theta}=3 k \theta / S(S+1) \\
& \lambda_{C}=3 k T_{C} / S(S+1)
\end{aligned}
$$

The theoretical dependences of the magnetization (2) for both cases are shown in Fig. 5 in comparison with the experimental ones measured in various external magnetic fields applied in the direction of easy magnetization. For the field $H=70 \mathrm{kOe}$ (Fig. 5c), the experimental dependence is presented using the magnetization isotherms given in [4]. When the parameter $\lambda_{\theta}$ corresponding to the paramagnetic Curie temperature (the solid red lines in Fig. 5) is used, good 
agreement with the experiment is achieved in the high-temperature region $T \geq 2.5 T_{C}$ and in the relatively large magnetic fields $H_{0} \geq 10 \mathrm{kOe}$. For the parameter $\lambda_{C}$, the mean field approximation does not give a satisfactory agreement with the experiment both below and above the ordering temperature in any magnetic field (the dushed blue lines in the Figures).
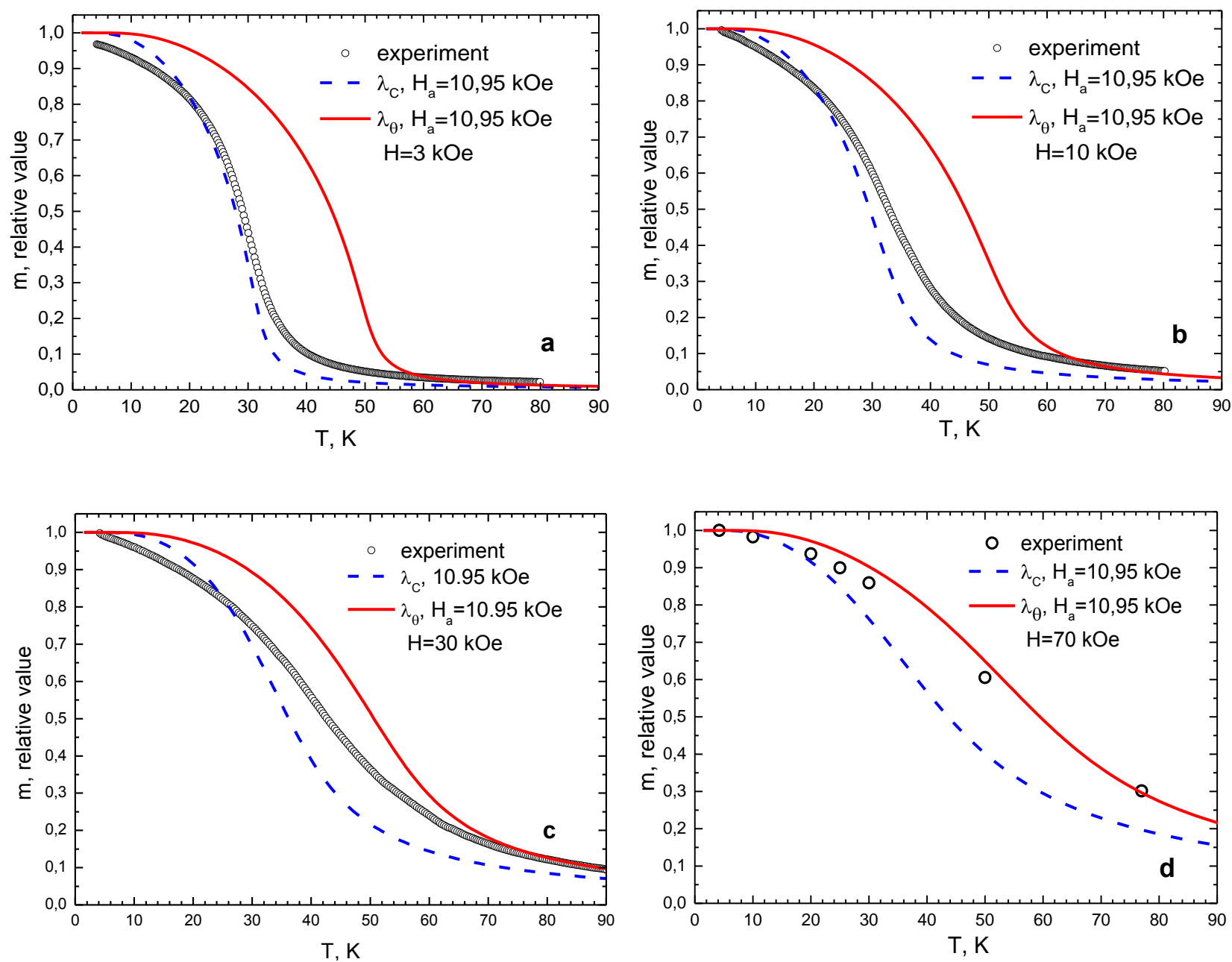

Fig. 5. Comparison of the theoretical and experimental temperature dependences of magnetization measured in different magnetic fields.

The significant difference between the magnetic ordering temperature $T_{C}$ and the paramagnetic Curie temperature $\theta$ is a sign either of the frustration of the exchange bonds or of the quasi-low-dimensional spatial distribution of the exchange interactions. For ferromagnetic ordering, the first reason is absent since the mutual orientations of the spins below the ordering temperature coincide with their orientations in the external magnetic field in the high-temperature limit. At the same time, the crystal structure of $\mathrm{PbABO}_{4}$ creates the prerequisites for the formation of a quasi-one-dimensional magnetic structure. The crystal structure of this crystal and its role in the formation of the magnetic structure of $\mathrm{PbMnBO}_{4}$ were considered in detail in [4]. The main fragments of the structure are linear $\mathrm{MnO}_{4}$ chains made up of edge-sharing $\mathrm{MnO}_{6}$ octahedra; the chains are linked by the $\mathrm{BO}_{3}$ and $\mathrm{PbO}_{4}$ groups. This peculiarity of the structure can lead to the fact that the exchange interaction between the $\mathrm{Mn}^{3+}$ ions in the chain is stronger than the interaction between them, which can lead to a difference between $T_{C}$ and $\theta$. With the strong difference between the intra- and interchain interactions, other features are observed in such structures: as a rule, the temperatures of the establishment of the long-range magnetic order are suffi- 
ciently low, and in the paramagnetic region, a broad peak of the specific heat is observed ${ }^{2}$ which is due to the establishment of short-range magnetic correlations in the chains. Similar features were observed in quasi-one-dimensional magnets formed from the ferromagnetic chains $\left(\mathrm{C}_{6} \mathrm{H}_{11} \mathrm{NH}_{3}\right) \mathrm{CuCl}_{3},\left(\mathrm{C}_{6} \mathrm{H}_{11} \mathrm{NH}_{3}\right) \mathrm{CuBr}_{3}$ [6, 7], $\mathrm{RbFeCl}_{3}$ [8] and $\mathrm{CsNiF}_{3}$ [9].

Currently, there is no consensus on the dimensionality of the magnetic structure of the crystals of the $\mathrm{PbABO}_{4}$ group with $\mathrm{A}=\mathrm{Fe}, \mathrm{Mn}$ and $\mathrm{Cr}$. In [2], these crystals are classified as quasi-low-dimensional magnets, since the authors found broad peaks of the magnetic susceptibility when studying the polycrystals with $\mathrm{A}=\mathrm{Fe}$ and $\mathrm{Cr}$. In addition, the study of polarized Raman scattering in the $\mathrm{PbFeBO}_{4}$ crystal shows [10] that the intrachain exchange interaction is dominant, which evidences the quasi-low-dimensional character of the magnetic structure of this antiferromagnet. The results of the calculation of the exchange parameters for this crystal [11] obtained by the density functional (DFT) method $J_{0} / k_{B}=-21 \mathrm{~K}$ (intra-) and $J_{2} / k_{B}=-3.9 \mathrm{~K}$ (inter-) also indicate the dominant character of the intrachain exchange.

However, our measurements [3] performed on the single-crystal $\mathrm{PbFeBO}_{4}$ show the absence of a broad peak of the magnetic susceptibility in the paramagnetic region; the temperature dependence of the susceptibility in the paramagnetic region follows the Curie-Weiss law. Most likely, the broad peak of the susceptibility observed in [2] is not due to the correlations of the short-range magnetic order but is rather induced by the contribution of the $\alpha-\mathrm{Fe}_{2} \mathrm{O}_{3}$ hematite phase present in the polycrystalline sample. In addition, the $\mathrm{PbFeBO}_{4}$ crystal has a relatively high Neel temperature $T_{N}=114 \mathrm{~K}$, which is not typical of most quasi-low-dimensional magnets. Based on these data, it is concluded [3] that the $\mathrm{PbFeBO}_{4}$ crystal is most likely a threedimensional antiferromagnet.

A similar situation is observed in the ferromagnet $\mathrm{PbMnBO}_{4}$. The DFT calculations of the exchange parameters for this crystal [11] also show the difference between the inter- and intrachain exchange parameters. However, there is no pronounced wide peak on the temperature dependence of the specific heat in the paramagnetic region which is smooth and well described by the lattice contribution (see the inset in Fig. 2).

Thus, one can summarize the following. All the crystals of the $\mathrm{PbABO}_{4}$ group with $\mathrm{A}=\mathrm{Fe}$, $\mathrm{Mn}$ and $\mathrm{Cr}$ have structural prerequisites for the formation of the quasi-one-dimensional magnetic structure. In these crystals, the exchange intrachain interactions are stronger than the interchain ones, which is qualitatively confirmed by the theoretical DFT calculations as well as in the experimental Raman studies of the $\mathrm{PbFeBO}_{4}$ crystal. However, in the crystals with $\mathrm{A}=\mathrm{Fe}$ and $\mathrm{Mn}$ this difference between the interactions is apparently not so great; and in these crystals, the temperature ranges for the formation of the short-range magnetic order are close enough to the corresponding temperatures of the magnetic phase transitions. As a result, in the paramagnetic regions of both crystals there are no pronounced maxima of the thermodynamic characteristics which are associated with the establishment of the short-range magnetic order. On the other hand, the difference between the intra- and interchain exchange interactions in $\mathrm{PbMnBO}_{4}$ leads to the fact that the paramagnetic Curie temperature $\theta \approx 49 \mathrm{~K}$ which is found from the hightemperature susceptibility and determined by the sum of both exchange interactions considerably exceeds the transition temperature $T_{C}$. And it is the difference in the exchange interactions which leads to the fact that the magnetic contribution to the specific heat is observed over a wider tem-

\footnotetext{
${ }^{2}$ In quasi-low-dimensional antiferromagnets, a broad peak of the magnetic susceptibility appears also in the same temperature region, but such a peak of the susceptibility is absent in quasi-low-dimensional ferromagnets.
} 
perature range above $T_{C}$ than in three-dimensional magnetic materials with isotropic exchange interaction.

A similar situation seems to be observed in $\mathrm{PbFeBO}_{4}$ where the difference between the intra- and interchain exchange interactions leads to the fact that the paramagnetic Néel temperature in its absolute value $(\approx 260 \mathrm{~K})$ is more than twice than that of $T_{N}$. But this difference between the exchange parameters is also insufficient to resolve the broad maximum of the susceptibility in the paramagnetic temperature range.

Apparently, only in $\mathrm{PbCrBO}_{4}$ the interaction between the chains is quite small as compared to the intrachain one, which leads both to the small value of the Néel temperature $T_{N}=8 \mathrm{~K}$ and the well pronounced maximum of the magnetic susceptibility at $T\left(\chi_{\max }\right) \approx 13 \mathrm{~K}$.

Having the experimental values of $T_{C}$ and $\theta$ for $\mathrm{PbMnBO}_{4}$ and using their theoretical expressions (3), one can determine the parameters of the spin Hamiltonian - both the exchange between the spins within the chain $J$ and the total interchain exchange $z^{\prime} J^{\prime}$ (for the structure $\mathrm{PbMBO}_{4}$, the number of the neighboring chains $z^{\prime}=4$ ). The single-ion anisotropy parameter $K$ used for the analysis of the temperature behavior of magnetization (2) is small compared both to the temperatures at which the determination of $\theta$ was carried out and the ordering temperature $T_{C}$, with the latter being increased by $4 \%$. Therefore, it was not taken into account when estimating the exchange parameters of the Hamiltonian (1).

The paramagnetic Curie temperature $\theta$ is obtained using the asymptotic limit $\mathrm{T} \rightarrow \infty$ in the expression for the relative magnetization (2) using the exchange field parameter for both exchange interactions in the Hamiltonian (1) [12]

$$
\lambda=\frac{k}{2} \sum_{n n} J_{i j}=k\left(J+\frac{z^{\prime}}{2} J^{\prime}\right),
$$

which gives

$$
\theta=\frac{S(S+1)}{6}\left(2 J+z^{\prime} J^{\prime}\right)
$$

An analytical expression for the temperature of the quasi-one-dimensional magnet ordering is obtained for the chain of classical spins $S=1$ in the mean field of neighboring chains in the continuum approximation of the Ginzburg-Landau field theory [13] and is used to describe the magnetic properties of $\mathrm{CsNiF}_{3}$ [14]. Applying the transition from the exchange integral in the system of classical spins of the unit length to the integral in the system of quantum spins $S$ $\left.J_{\|}\left(J_{\perp}\right)=S^{2} J^{\prime} J^{\prime}\right)$ [15] and using the second term (4) as the coefficient of the interchain exchange field, we obtain an expression for the temperature of ordering

$$
T_{C} \cong S^{2} \sqrt{\frac{z^{\prime}}{3} J^{\prime} J}
$$

Substituting the experimental values of $T_{C}$ and $\theta$ in (5) and (6), one obtains an estimate of the exchange parameters of the Hamiltonian (1):

$$
J \cong 20.2 K, J^{\prime} \cong 2.1 K
$$

The obtained value of the exchange within the chain almost coincides with the result of describing the susceptibility of $\mathrm{PbMnBO}_{4}$ as the susceptibility for the one-dimensional chain [2] 
obtained using the classical Fisher approach [15] $\left(J_{\mathrm{S}}=11 \mathrm{~K}\right.$ for the Hamiltonian $\left.H=-2 J_{S} \sum_{i} \mathbf{S}_{i} \mathbf{S}_{i+1}\right)$. Thus, the parameters of the spin Hamiltonian (1) obtained from the experimental values of $T_{C}$ and $\theta$ confirm the chain character of the spatial distribution of the exchanges in $\mathrm{PbMnBO}_{4}$. The theoretical DFT calculation of the exchange parameters for this magnet [11] yields $J=37 \mathrm{~K}, J_{1}^{\prime}=0.5 \mathrm{~K}$ and $J_{2}^{\prime}=3.0 \mathrm{~K}$ where $J_{1}^{\prime}$ and $J_{2}^{\prime}$ describe the interchain exchange interactions with $z_{1}^{\prime}=4$ and $z_{2}^{\prime}=8$, respectively. These values of the exchange parameters can only be regarded as a qualitative confirmation of the quasi-one-dimensional character of the magnetic structure of $\mathrm{PbMnBO}_{4}$ since the estimate of the paramagnetic Curie temperature using these parameters is twice as high as the experimental value of $\theta$.

The temperature interval in which the Ginzburg-Landau field theory is applicable was obtained in [16]; in the notation of the Hamiltonian (1) this interval has the form

$$
S^{2} J>T>S^{2} \sqrt{\frac{z^{\prime}}{3} J^{\prime} J}=T_{C} .
$$

Thus, the upper limit of this interval $\mathrm{T} \approx 80 \mathrm{~K}$ quantitatively corresponds to the temperature above which the mean field approximation with the parameter $\lambda_{\theta}$ well describes the experimental temperature dependences of magnetization measured in the magnetic fields $H_{0} \geq 10 \mathrm{kOe}$.

At low temperatures $T<T_{C}$, the magnetization of $\mathrm{PbMnBO}_{4}$ rapidly decreases with the increasing temperature. The same decrease is observed for the sublattice magnetization in $\mathrm{CsNiF}_{3}$ [17]. Such a behavior can be explained by the existence in the ordered phase of nonlinear excitations of the sin-Gordon type [18] in a chain magnet with small anisotropy in the easy plane of magnetization. In $\mathrm{PbMnBO}_{4}$, the value of anisotropy is much smaller than the above-mentioned temperature of the magnetic measurements, which provides the conditions for the existence of such excitations decreasing the magnetization.

The calculation of the temperature dependence of the magnetic contribution to the specific heat in the external field can be performed both below and above $T_{C}$ similarly [19-21]:

$$
C_{m}=\frac{d E_{m}}{d T}=\frac{d}{d T} H_{e f f} M
$$

where $E_{m}$ is the magnetic energy and $H_{\text {eff }}$ is determined in (2). In this case, both the temperature dependence of magnetization (2) calculated in the framework of the mean field approximation and the experimentally measured dependence (Fig. 1) can be used for the calculation. In both cases, the exchange field is written as $H_{E}=\lambda M / g^{2} \mu_{B}{ }^{2}$. The substitution of this expression in (8) gives

$$
C_{m}=\left(H_{0}+\frac{2 \lambda M}{g^{2} \mu_{B}^{2}}\right) \frac{d}{d T} M
$$

The 1/2 multiplier for the exchange term of the effective field in [18-20] should be taken into account in the transition from the Hamiltonian of the exchange interaction to the exchange field $H_{E 1,2}$ from each exchange coupling

$$
J \mathbf{S}_{1} \mathbf{S}_{2} \rightarrow g \mu_{B}\left(\mathbf{H}_{E 2} \mathbf{S}_{1}+\mathbf{H}_{E 1} \mathbf{S}_{2}\right), \quad \mathbf{H}_{E 1}=\mathbf{H}_{E 2}=\frac{\lambda\langle\mathbf{S}\rangle}{g \mu_{B}},
$$


which is necessary to avoid doubling the total exchange energy of the spins. Taking this into account leads to the appearance of this factor for a number of magnetic neighbors when the parameter $\lambda$ and exchange integrals (4) are compared.

Fig. 6 shows the results of the comparison of the theoretical dependences of the magnetic contribution to the specific heat (9) with the experimental ones measured in the magnetic fields of 3 and $30 \mathrm{kOe}$. The figures show the results of the calculations using both the experimental dependences of magnetization (the red lines) and mean field approximation (the blue lines). In the Figure, the dotted and solid lines correspond to the use in (9) of either $\lambda_{C}$ or $\lambda_{\theta}$, respectively, as an exchange parameter. The comparison shows that the best agreement with the experiment is obtained in the strong magnetic field $H_{0} \geq 30 \mathrm{kOe}$ when using both the experimental temperature dependence of magnetization and mean field coefficient $\lambda_{\theta}$. Moreover, in this case a good description of the experimental data is obtained not only in the high-temperature region, but also in the vicinity of $T_{C}$. In the weak magnetic field $H=3 \mathrm{kOe}$, the agreement with the experimental data is unsatisfactory for any methods of calculation of the theoretical dependence: neither for the mean field approximation nor for the experimental dependence $M(T)$. Apparently, in the weak magnetic fields, in the expression for the effective field $H_{\text {eff }}$ in (8) it is necessary to take into account the magnetic anisotropy comparable with $H_{0}$.
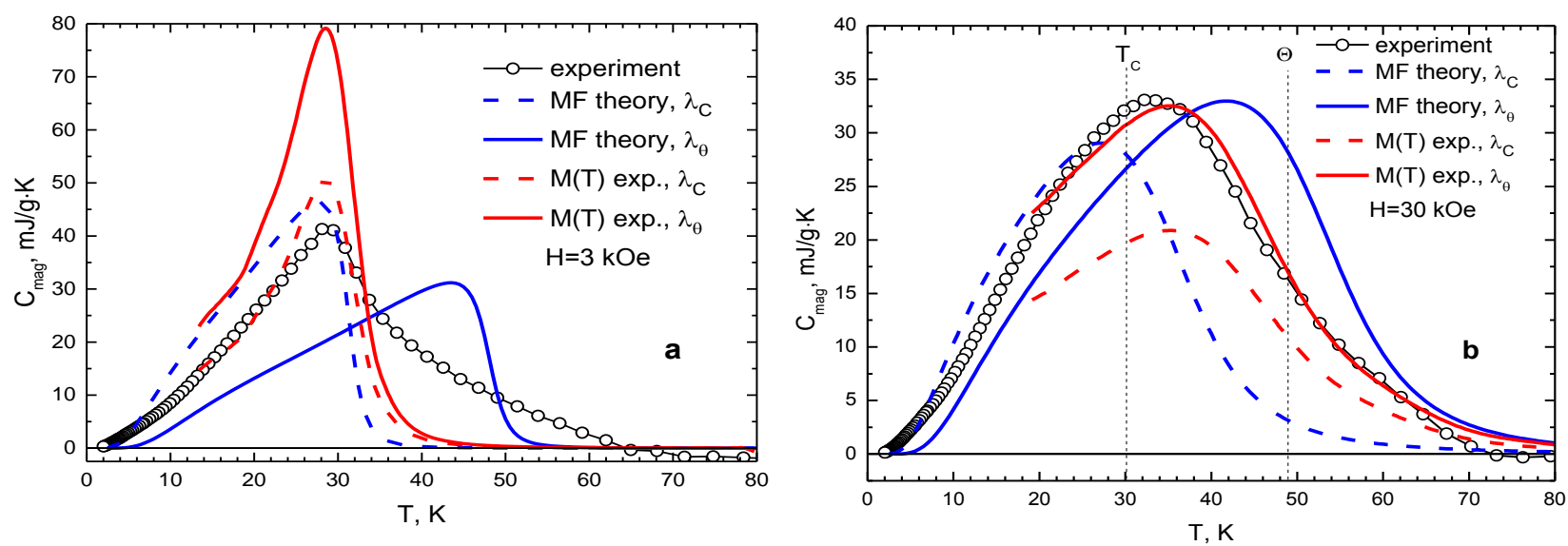

Fig. 6. Temperature dependences of the magnetic contributions to the specific heat measured in magnetic fields of $3 \mathrm{kOe}$ (a) and $30 \mathrm{kOe}$ (b). Points - experiment, lines - calculations (see text).

Although the intrachain exchange in $\mathrm{PbMnBO}_{4}$ exceeds the interchain one by an order of magnitude the complete interchain exchange $z^{\prime} J^{\prime}$ is comparable to $2 J$ which leads to the temperature of ordering $T_{C}$ which differs by a factor of 1.5 from the paramagnetic temperature $\theta$. In addition, because of the relatively small difference between the intra- and interchain interactions in this crystal, the influence of the short-range magnetic order in the chains has its own peculiarities.

In classical quasi-one-dimensional magnetic materials in which the ratio of exchanges is $J^{\prime} / J \sim 10^{-2}-10^{-3}$, the wide maximum of the specific heat due to the formation of the short-range magnetic order in the chains is in the paramagnetic region far from the temperature of the magnetic phase transition and is clearly visible, as in the systems already mentioned [6-9]. In the other limiting case of three-dimensional magnetic materials with an isotropic exchange interaction, the magnetic contribution to the specific heat does not fall to zero at the temperature of the phase 
transition, and a "tail" is observed in a certain temperature interval (usually small) above Tc, due to the disruption of the 3D short-range magnetic order.

The $\mathrm{PbMnBO}_{4}$ crystal refers to the intermediate case when there is only a 5-fold difference between the complete inter- $z^{\prime} J$ and intrachain $2 J$ exchanges. Therefore, the wide peak of the magnetic heat capacity due to the short-range order in the chains is superimposed on this "tail" and is poorly discernable against its background.

To illustrate this effect, we subtracted the contribution of a one-dimensional Heisenberg chain for $S=1 / 2$ [22] from the experimental temperature dependence of the magnetic heat capacity for $H=0$. In Fig. 7 this contribution is constructed for $J=20 \mathrm{~K}$ and is shown by the dashed line. Subtraction is meaningful at temperatures $T>T_{C}$ since there is a long-range magnetic order in the ordered region. The result of the subtraction marked by the red solid line in the figure is the contribution of 3D correlations and above $T_{C}$ its form corresponds to the usual behavior of the magnetic heat capacity for magnets with isotropic exchange.

Yet, such an illustration is only qualitative since here, the contribution of one-dimensional chains with $S=1 / 2$ is used. However, it clearly demonstrates that in quasi-one-dimensional magnets with a relatively small difference in intra- and interchain interactions, the short-range order correlations in the chains do not lead to the formation of the well-discernible broad maximum of the specific heat in the paramagnetic region, but increase the temperature interval above $T_{C}$ in which an appreciable magnetic contribution to the heat capacity is observed. This allows us to classify $\mathrm{PbMnBO}_{4}$ as a chain magnet with an intermediate magnetic dimension between the quasi-one-dimensional and three-dimensional ones.

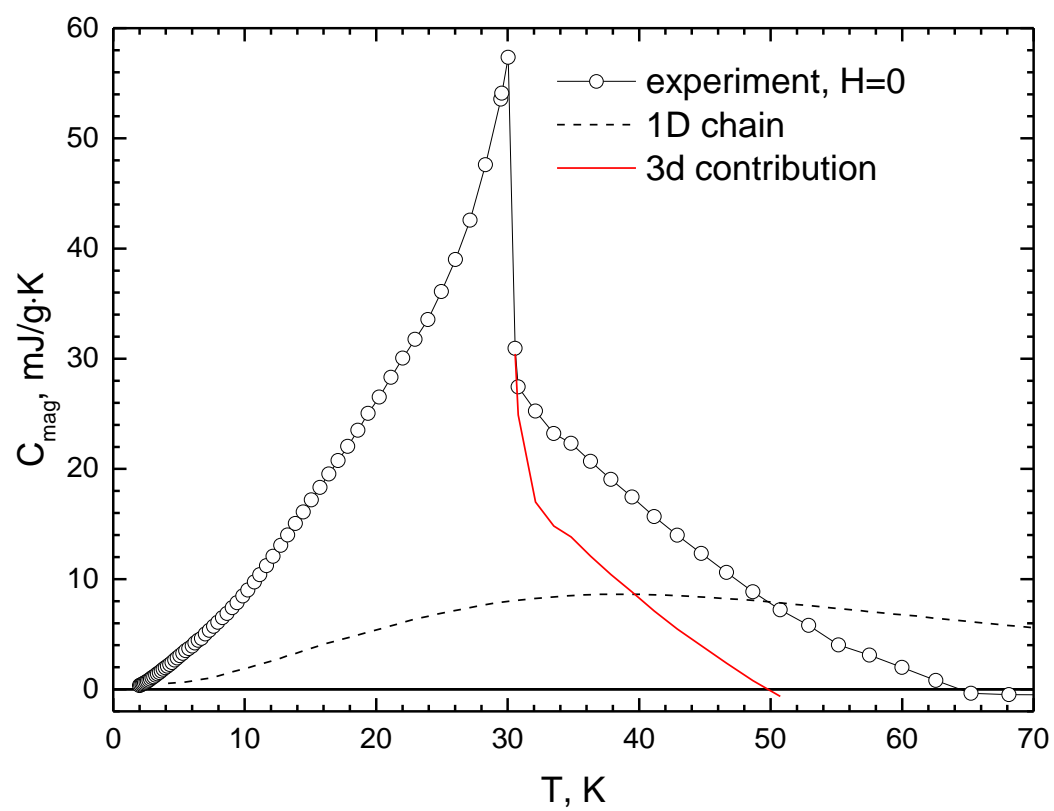

Fig. 7. Magnetic contribution to the specific heat for $\mathrm{H}=0$ as a sum of $1 \mathrm{D}$ and $3 \mathrm{D}$ contributions (see text).

In this case, the correct application both of the quasi-one-dimensional approach and the mean field approximation allows one to correctly quantitatively describe the experimental data within the framework of the relatively simple Hamiltonian (1). Nevertheless, it should be noted that the Hamiltonian used is a very rough approximation. It does not take into account a number of features of the crystal structure, namely, the nonequivalence both of the magnetic chains and environments of the spins in the chains. These features lead to the rotation of the local anisotropy 
axes for $\mathrm{Mn}^{3+}$ ions both in the neighboring positions in the chain and in the neighboring chains. Moreover, the anisotropy of the paramagnetic Curie temperature indicates the presence of additional anisotropy mechanisms apart from the single-ion mechanism. Therefore, its description within the framework of Hamiltonian (1) should be considered only as the first approximation.

\section{Conclusion.}

In the $\mathrm{PbMnBO}_{4}$ single crystal, the temperature dependences of the magnetization and specific heat were measured in the external magnetic fields of 3,10, and $30 \mathrm{kOe}$. The magnetic contribution to the specific heat measured in the zero magnetic field shows a sharp jump at the Curie temperature $T_{C}=30.3 \mathrm{~K}$. When measured in the magnetic field, this jump is smeared into a smooth hump which broadens and shifts towards higher temperatures with the increasing magnetic field. For all the magnetic fields, the calculated transition entropy corresponds to the value $R \ln (2 S+1)$, confirming the purely magnetic character of the transition. It was found that even in the zero magnetic field, the temperature region in which the magnetic contribution to the heat capacity is maintained above $T_{\mathrm{C}}$ was significant, the magnetic contribution to the specific heat associated with the phase transition was observed up to $\sim 65 \mathrm{~K}$, and in the magnetic field of 30 kOe this contribution disappeared only at temperatures above $70 \mathrm{~K}$.

The temperature and field dependences of the magnetization also indicate that in the strong magnetic field the magnetization decreases with the increasing temperature much slower than in classical three-dimensional ferromagnets, and the field dependences of the magnetization remain nonlinear up to the temperatures exceeding $T_{\mathrm{C}}$ more than twice. The experimental temperature dependences of the magnetization were compared with the theoretical ones calculated in the mean field approximation. The mean field coefficient $\lambda$ used in this approximation was calculated either using the Curie temperature $T_{C}$ or paramagnetic Curie temperature $\theta=49 \mathrm{~K}$ obtained from the high-temperature part of the magnetic susceptibility at $T>175 \mathrm{~K}$. It is established that the mean field approximation well describes the experimental data only in the hightemperature region and in relatively large magnetic fields with the exchange parameter $\lambda_{\theta}$.

It is assumed that in $\mathrm{PbMnBO}_{4}$, the difference between $T_{C}$ and $\theta$ and the wide temperature region above $T_{C}$ in where the magnetic contribution to the specific heat is significant are due to the quasi-one-dimensional character of the magnetic structure of this ferromagnet. Using both the value of $T_{C}$ from the Ginzburg-Landau field theory and the $\theta$ value, the intrachain exchange interaction parameter $2 J \approx 40.4 \mathrm{~K}$ and total interchain exchange interaction parameter $z^{\prime} J \approx 8.8 \mathrm{~K}$ are determined, with $z^{\prime}=4$ being the number of the neighboring chains. The estimation shows that the Ginzburg-Landau field theory describing the quasi-one-dimensional behavior of $\mathrm{PbMnBO}_{4}$ is well applicable in the temperature range from $T_{C}$ to $T=S^{2} J \approx 80 \mathrm{~K}$. Above this temperature, the mean field approximation with the parameter $\lambda_{\theta}$ well describes the experimental temperature dependences of the magnetization in the strong magnetic field and the specific heat is determined by the lattice contribution.

The established difference in the parameters of the intra- and interchain exchange interactions is much smaller than in the traditional quasi-one-dimensional magnets. As a result, the short-range order correlations in the chains do not lead to the formation of the well-discernible broad maximum of the specific heat in the paramagnetic region but increase the temperature interval above $T_{C}$ in which the magnetic contribution to the heat capacity is significant. 


\section{Acknowledgments}

This work was supported by the Russian Foundation for Basic Research, Project no. 16-0200563. The authors thank V. Zinenko and V. Sosnin for the fruitful and useful discussions.

\section{References.}

[1] H. Park and J. Barbier, Acta Crystallogr. E57 (2001) 82.

[2] H. Park, R. Lam, J.E. Greedan and J. Barbier, Chem. Mater. 15 (2003) 1703.

[3] A. Pankrats, K. Sablina, D. Velikanov, A. Vorotynov, O. Bayukov, A. Eremin, M. Molokeev, S. Popkov and A. Krasikov, JMMM 353 (2014) 23.

[4] A. Pankrats, K. Sablina, M. Eremin, A. Balaev, M. Kolkov, V. Tugarinov and A. Bovina, JMMM 414 (2016) 82.

[5] S.V. Vonsovsky, Magnetism, Moscow: Nauka, 1971.

[6] H.A. Groenendijk, H.W.J. Blote, A.J. van Duyneveldt, R.M. Gaura, C.P. Landee and R.D. Willett, Physica B: Condensed Matter 106 (1981) 47.

[7] K. Kopinga, T. Delica and H. Leschke, Phys. Rev. B 40 (1989) 7239.

[8] P.A. Montano, E. Cohen, H. Shechter and J. Makovsky, Phys. Rev. B 7 (1973) 1180.

[9] J.V. Lebesque, J. Snell and J.J. Smit, Solid State Communications 13 (1973) 371.

[10] M.A. Prosnikov, A.N. Smirnov, V.Yu. Davydov, K.A. Sablina and R.V. Pisarev, J. Phys.: Condens. Matter 29 (2017) 025808.

[11] H.-J. Koo and M.-H. Whangbo, Solid State Communications 149 (2009) 602.

[12] S.N. Martynov, JMMM 398 (2016) 121.

[13] D.J. Scalapino, Y. Imry and P. Pincus, Phys. Rev. B 11 (1975) 2042.

[14] A.R. McGurn, D.J. Scalapino and Y. Imry, Solid State Communications 11 (1975) 305.

[15] M.E. Fisher, Am. J. Phys. 32 (1964) 343.

[16] A.R. McGurn and D.J. Scalapino, Phys. Rev. B 11 (1975) 2552.

[17] C. Dipas and J.-P. Renard, J. Phys. C 10 (1977) 5057.

[18] T. Delica, W.J.M. de Jorge, K. Kopinga, H. Leschke and H.J. Mikeska, Phys. Rev. B 44 (1991) 11773.

[19] D.C. Mattis, The Theory of Magnetism, Harper \& Row Publishers, New York, 1965.

[20] J.S. Smart, Effective Field Theories of Magnetism, Saunders W.B. Company, PhiladelphiaLondon, 1966.

[21] A. Tari, The Specific Heat of Matter at Low Temperatures, World Scientific, 2003.

[22] J.C. Bonner and M.E. Fisher, Phys. Rev. 135 (1964) A640. 